\title{
Small molecule MIRA-1 induces in vitro and in vivo anti-myeloma activity and synergizes with current anti-myeloma agents
}

\author{
M N Saha ${ }^{1,2,5}$, Y Chen ${ }^{1,2,3,5}$, M-H Chen ${ }^{1,2}$, G Chen $^{3}$ and H Chang ${ }^{\star, 1,2,3,4}$ \\ ${ }^{1}$ Division of Molecular and Cellular Biology, Toronto General Research Institute, Toronto, Ontario, Canada; ${ }^{2}$ Department of \\ Laboratory Medicine \& Pathobiology, University of Toronto, Toronto, Ontario, Canada; ${ }^{3}$ Department of Hematology, The First \\ Affiliated Hospital of Nanchang University, Nanchang, China and ${ }^{4}$ Department of Laboratory Hematology and Medical Oncology, \\ University Health Network, Toronto, Ontario, Canada
}

Background: Small molecule MIRA-1 induced mutant p53-dependent apoptosis in several types of solid tumours. However, anti-tumour activity of MIRA-1 in haematological malignancies including multiple myeloma (MM) is unknown. In this study, we evaluated the effect of MIRA-1 in MM.

Methods: We examined the anti-tumour activity of MIRA-1 alone or in combination with current anti-myeloma agents in a panel of MM cell lines, primary MM samples, and in a mouse xenograft model of MM.

Results: MIRA-1 treatment resulted in the inhibition of viability, colony formation, and migration and increase in apoptosis of MM cells irrespective of p53 status accompanied by upregulation of Puma and Bax and downregulation of Mcl-1 and c-Myc. Genetic knockdown of p53 did not abrogate apoptotic response of MIRA-1. MIRA-1 triggered activation of PERK and IRE- $\alpha$ leading to splicing of XBP1 indicating an association of endoplasmic reticulum stress response. Furthermore, combined treatment of MIRA-1 with dexamethasone, doxorubicin or velcade displayed synergistic response in MM cells. Importantly, MIRA-1 alone or in combination with dexamethasone retarded tumour growth and prolonged survival without showing any untoward toxicity in the mice bearing MM tumour.

Conclusions: Our data provide the preclinical framework for clinical evaluation of MIRA-1 as a novel therapeutic agent to improve patient outcome in MM.

Multiple myeloma (MM) is an incurable haematological malignancy that arises from the dysregulated proliferation of plasma cells. The use of novel anti-myeloma agents (such as thalidomide, lenalidomide, and velcade/bortezomib), alone or in combination with conventional chemotherapy (e.g., dexamethasone and doxorubicin) has improved the clinical outcome of patients. However, the development of drug resistance is universal and associated with disease progression (Anderson, 2011; Dimopoulos et al, 2011). Significant advances in both basic and translational research have enhanced understanding of myeloma pathogenesis and prompted to test new and more effective molecules as therapeutic targets (Mitsiades et al, 2009; Anderson, 2011).
Restoring function of $\mathrm{p} 53$, a tumour suppressor protein, has long been considered an attractive anti-cancer therapeutic approach. The prevalence of TP53 mutations differs considerably between tumour types and stages of cancer, and $\sim 50 \%$ of all tumours present mutations. In MM, mutations of the TP53 gene is rarely detected at diagnosis, although it becomes more frequent in advanced disease and human MM cell lines (Mazars et al, 1992; Chang et al, 2005; Saha et al, 2010a). Contrary to many other types of cancer, it is not yet known if the TP53 gene has an essential role in the pathogenesis of this disease. Despite successful identification and preclinical studies of the small molecules targeting p53 such as, nutlin, RITA, and PRIMA-1 ${ }^{\text {Met }}$, further evaluation for additional

\footnotetext{
*Correspondence: Professor H Chang; E-mail: Hong.Chang@uhn.on.ca
}

${ }^{5}$ These authors contributed equally to this work. 
p53 reactivating molecules especially which can target mutant p53 remains a highly important task (Bykov et al, 2002; Issaeva et al, 2004; Vassilev et al, 2004; Wiman, 2006; Wang and El-Deiry, 2008; Saha et al, 2013a).

Pharmacological screening of a diverse set of low molecular weight compounds led to the identification of a novel class of mutant p53 reactivating molecules, MIRA-1, a maleimide derivative (Figure 1A) (Bykov et al, 2005). MIRA-1 is structurally distinct from the previously described mutant p53-targeting compounds PRIMA-1, identified in the similar screening by the same groups. Studies using solid tumour cell models showed that MIRA-1 induces apoptosis via restoration of p53-dependent transcriptional transactivation. Importantly, MIRA-1 induces cell death with a potency that is even higher than that of PRIMA-1 (Bykov et al, 2005). To date, anti-tumour activity of MIRA-1 in haematological malignancies including MM is undefined. In the present study, we examined anti-myeloma activity of MIRA-1 using both in vitro and in vivo model systems. Our studies show that MIRA-1 is a potent small molecule which can kill MM cells harbouring wild-type or mutant p53.

\section{MATERIALS AND METHODS}

Cell culture. MM.1S, NCI-H929 (H929), RPMI-8226 (8226), LP1, and U266 human MM cell lines were obtained from American Type Culture Collection. All MM cell lines were cultured and maintained as described previously (Saha et al, 2010b,c,d). Primary MM samples were obtained from bone marrow aspirates of newly diagnosed patients. Bone marrow mononuclear cells (BMMNCs) were separated using Ficoll-Hipaque density sedimentation. Primary CD138 + plasma cells were purified using negative selection, as in previous studies (Saha et al, 2010b). Blood samples collected from healthy volunteers were processed by FicollHipaque gradient to obtain peripheral blood mononuclear cells (PBMCs). All procedures were performed with Institutional Review Board-approved (University Health Network, Toronto) protocols and informed consent, and in accordance with the declaration of Helsinki.

Reagents. MIRA-1 was obtained from Tocris Bioscience (Bristol, UK). For in vitro experiments, MIRA-1 was dissolved in dimethyl sulfoxide (DMSO) to create a $100 \mathrm{mmoll}^{-1}$ stock solution and stored at $-20^{\circ} \mathrm{C}$. Dexamethasone and doxorubicin were obtained from Biovision (Milpitas, CA, USA) and bortezomib was obtained from Selleck Chemicals (Houston, TX, USA).

Cell viability assay. The growth inhibitory effects of MIRA-1 in MM cell lines, primary MM samples, BMMNCs, and PBMCs were assessed by measuring 3-(4,5-dimethylthiazol-2-yl)-2,5-diphenyl tetrasodium bromide (MTT; Sigma-Aldrich, St Louis, MO, USA) dye absorbance, as described previously (Saha et al, 2010b, c, d; Saha et al, 2012).

Detection of apoptosis. Apoptosis detection was performed with the Annexin V-propidium iodide (PI) detection kit (Abcam, Cambridge, MA, USA). Multiple myeloma cells were exposed with various concentrations of MIRA-1 for $48 \mathrm{~h}$ and apoptotic cells were analysed on a BD FACS Canto II (BD Biosciences, San Jose, CA, USA) using FACSDiva (BD Biosciences) as described by us previously (Saha et al, 2013b).

Colony forming and migration assay. Colony formation and migration assay was performed as described previously (Saha et al, 2013b). For colony-forming assays, after treatment with MIRA-1, MM cells $\left(5 \times 10^{4}\right.$ cells $\left.\mathrm{ml}^{-1}\right)$ were plated into methylcellulose medium in triplicate for 14 days. Multiple myeloma cell colonies
A

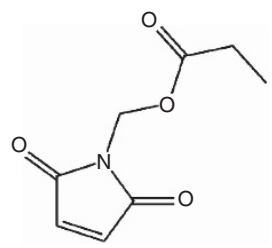

Chemical name: 1-[1-Oxopropoxy)methyl]1 -pyrrole-2,5-dione

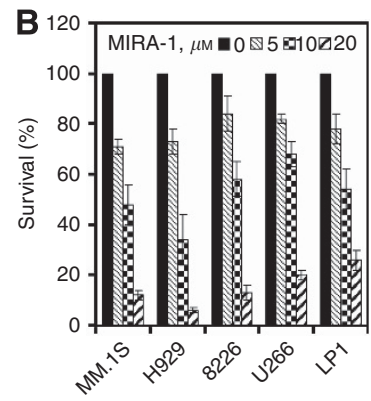

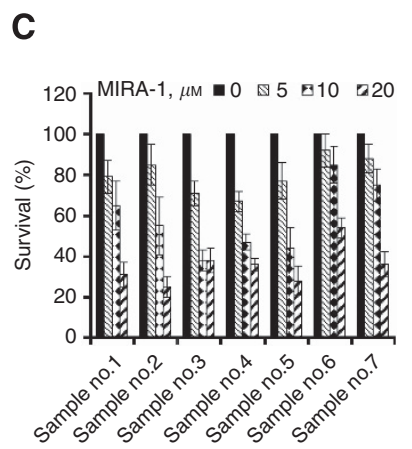
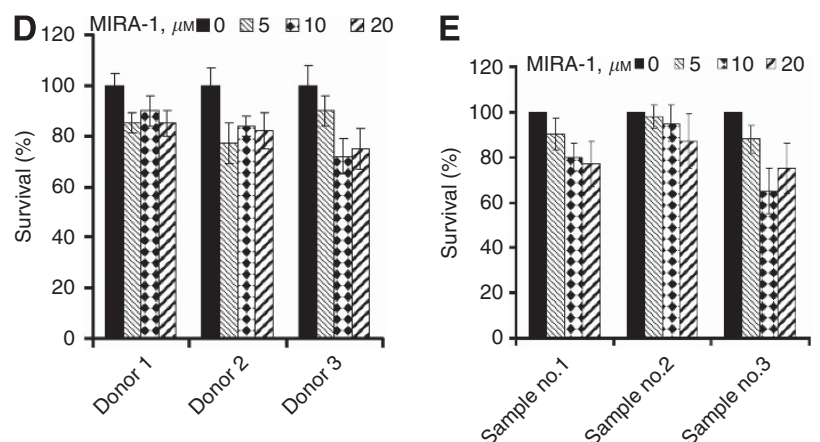

Figure 1. MIRA-1 demonstrated potent anti-myeloma activity in vitro. (A) Chemical structures of MIRA-1; Chemical name: 1-[(1Oxopropoxy)methyl]-1 $\mathrm{H}$-pyrrole-2,5-dione. Molecular formula: $\mathrm{C}_{8} \mathrm{H}_{9} \mathrm{NO}_{4}$ (B-E). (B) MM cell lines expressing wild-type p53 (MM.1S, H929) or mutant p53 (LP1, U266, and 8226) were incubated with MIRA-1 $\left(0-20 \mu \mathrm{moll}{ }^{-1}\right)$, and viability was determined at $48 \mathrm{~h}$ using MTT assay. (C) MIRA-1-induced cytotoxicity in primary MM samples from newly diagnosed MM patients. Myeloma cells isolated from the bone marrow via CD138 + selection were cultured with MIRA-1 (0-20 $\left.\mu \mathrm{moll}^{-1}\right)$, and viability was assessed by MTT at $48 \mathrm{~h}$. (D) PBMCs and (E) BMMNCs were treated similarly with MIRA-1 and cyototoxicity was assessed by MTT assay. Viability of the cells was expressed as percentage of the DMSO-treated control. Data represents means ( \pm s.d.) of triplicate cultures. 
that consist of $>40$ cells were counted under an inverted microscope. For studying migration, MM cells treated with MIRA-1 or DMSO were placed in 24-well Transwell insert chambers ( $8 \mu \mathrm{M}$ insert, Costar, Corning Inc., NY, USA) according to the manufacturer's instruction (Saha et al, 2013b). Migration was quantified by counting the number of cells on the lower surface of the filters. Results are expressed as mean number of migrating cells done in triplicates.

Transfection. Small interfering RNA (siRNA) of p53 and scrambled siRNA were purchased from Invitrogen (Carlsbad, CA, USA). siRNA was transfected into MM.1S or 8226 cells by electroporation using a Nucleofector Kit (Amaxa Biosystems, Koeln, Germany). Following transfection, cells were treated with MIRA-1 and the effect of apoptosis induction by MIRA-1 following knockdown of p53 was analysed by flow cytometry assay (Saha et al, 2012; Saha et al, 2013b).

Western blotting. MIRA-1-treated MM cells were harvested to isolate proteins. Whole cell lysates were subjected to sodium dodecyl sulfate-polyacrylamide gel electrophoresis and transferred to PVDF membrane (Millipore, Billerica, MA, USA), as described previously (Saha et al, 2010b,c,d; Saha et al, 2012; Saha et al, 2013b). Immunoblotting was performed using specific antibodies: anti-p53 (DO-7) (Sigma-Aldrich); Mcl-1, caspase-3, and PARP (Cell Signaling Technologies, Beverly, MA, USA); Puma, Bax, and c-Myc (Signalway Antibody, College Park, MD, USA); XBP1 and p-PERK (Thr 981) (Santa Cruz Biotechnology, Santa Cruz, CA, USA). Goat anti-mouse and anti-rabbit secondary antibodies conjugated to horseradish peroxidase were purchased from Cell Signaling and Santa Cruz Biotechnology, respectively. Blots were reprobed with anti- $\beta$ actin antibody (Signalway Antibody) for equal protein controls.

MM xenograft murine model. Severe combined immunodefficient (SCID) mice (OCI, Toronto, Canada) were monitored in the Animal Research Facility at University Health Network and subjected to studies according to the protocols approved by Animal Ethics Committee. $3 \times 10^{7} 8226$ cells mixed with matrigel (BD Biosciences) were inoculated subcutaneously into the right flank of 6-week-old SCID mice. When tumours were measurable, mice were treated with intraperitoneal (i.p.) injection of vehicle control $(0.9 \% \mathrm{NaCl})$ or MIRA-1 $\left(10 \mathrm{mg} \mathrm{kg}^{-1}\right)$ every alternate day for 2 weeks ( $n=5$ per group). In another set of experiments, four groups of mice ( $n=5$ per group) injected with 8226 cells were similarly treated with either MIRA-1 alone $\left(5 \mathrm{mg} \mathrm{kg}^{-1}\right)$, or dexamethasone alone $\left(1 \mathrm{mg} \mathrm{kg}^{-1}\right)$, or MIRA-1 in combination with dexamethasone, or vehicle. Mice were monitored for body weight, tumour size was measured every alternate days and tumour volume was calculated with the formula: $V=0.5\left(a \times b^{2}\right)$, where ' $a$ ' is the long diameter of the tumour and ' $b$ ' is the short diameter of the tumour. Mice were killed when the tumour reached $>1.5 \mathrm{~cm}$ or if mice appeared moribund to prevent unnecessary morbidity. Survival was evaluated from the first day of the treatment until death or an event occurs.

Statistical analysis. The combined effect of the drugs was analysed by CalcuSyn (Biosoft, Cambridge, UK), a software program based on the Chou-Talalay method, as described by us previously (Saha et al, 2010b,c,d; Saha et al, 2012; Saha et al, $2013 b)$. The equation for calculating combination index (CI) was: $\mathrm{CI}=(\mathrm{D}) 1 /(\mathrm{D} x) 1+(\mathrm{D}) 2 /(\mathrm{D} x) 2+(\mathrm{D}) 1(\mathrm{D}) 2 /(\mathrm{D} x) 1(\mathrm{D} x) 2$, where (D) 1 and (D) 2 are the doses of drug 1 and drug 2 that have $x$ effect when used in combination and (Dx) 1 and $(D x) 2$ are the doses of drug 1 and drug 2 that have the same $x$ effect when used alone (Saha et al, 2013b). A CI $<1.0$ is indicative of a synergistic effect. In the murine xenograft studies, survival of mice between the two treatment groups was determined by the Kaplan-Meier test using the log-rank analyses to compare the mean overall mouse survival with $95 \%$ confidence intervals. Statistical significance levels in different experiments were determined by two-tailed $t$-test analysis. The minimal level of significance was $P<0.05$.

\section{RESULTS}

MIRA-1 inhibited myeloma cell growth and induced apoptosis in vitro. MIRA-1 was previously reported to kill cancer cells of various solid tumours with mutant p53 (Bykov et al, 2005). To evaluate its activity against MM models, we studied a panel of MM cell lines with varying p53 status to determine sensitivity to MIRA-1. Wild-type p53 MM cells (MM.1S, H929) exhibited IC $_{50}$ values in the $7.5-10 \mu \mathrm{moll}^{-1}$ range, whereas mutant p53 cells (LP1, U266, 8226, OPM2) had $\mathrm{IC}_{50}$ values from 10 to $12.5 \mu \mathrm{moll}^{-1}$ (Figure 1B). These data suggested that cytotoxic response of MIRA-1 in MM cells is irrespective of p53 status.

To further assess the potential of MIRA-1 as a novel anti-myeloma agent, primary cells derived from seven newly diagnosed MM patients bone marrows were treated with vehicle control or escalating doses of MIRA-1 for $48 \mathrm{~h}$. Cells were then examined for viability by MTT assay. A significant decrease in the viability of MM cells was shown in all the samples with almost similar $\mathrm{IC}_{50}$ range as observed for $\mathrm{MM}$ cell lines (Figure 1C). Although there was some toxicity detected in the normal haematopoietic cells (PBMCs or BMMNCs) (Figure 1D and E), this was proportionately much less than the effect observed in cell lines or in primary MM samples.

To determine whether the reduction of cell viability was accompanied by apoptosis induction by MIRA-1, we performed Annexin $\mathrm{V}$ staining in combination with PI. MIRA-1 $\left(10 \mu \mathrm{moll}^{-1}\right)$ induced more than $50 \%-80 \%$ cell death in MM.1S and H929 cells harbouring wild-type p53. Although the mutant p53 cell models showed $32-45 \%$ cell death at similar concentration, $>80 \%$ cells were killed at $20 \mu \mathrm{moll}^{-1}$ MIRA-1 (Figure 2A). In consistence with the viability data, these results also suggest that MIRA-1 induces apoptosis in MM cells irrespective of p53 functional status.

MIRA-1 inhibits colony formation and migration of MM cells. Having shown the effect of MIRA-1 on viability and apoptosis, we next examine the effect of MIRA-1 on the colonyforming capability of MM cells. Treatment of MM cells with MIRA-1 significantly inhibits colony formation abilities (Figure 2B, $P<0.05)$ in MM.1S and LP1 cells. Because we found a significant inhibition of colony formation by MIRA-1, we proceeded to determine if MIRA-1 could inhibit the migration of MM cells. Migration of MM.1S and LP1 cells treated with MIRA-1 at 5 and $10 \mu \mathrm{moll}^{-1}$, respectively, was significantly lower as compared with the DMSO-treated control (Figure 2C, $P<0.05$ ). This difference was not due to cytotoxicity as cells treated in parallel and analysed via MTT showed $\sim 60 \%$ viability at this dose. These findings suggest that MIRA-1 suppressed the clonogenic and migratory potential of MM cells to form colonies and migrate into cells.

MIRA-1 modulated the expression of the members of $\mathrm{Bcl} 2$ family proteins in a time and dose-dependent manner. Knowing that MIRA-1 acts through p53 signalling pathway, we examined protein expression of p53 and its downstream targets by western blots. Results showed activation of caspase- 3 and PARP in MM.1S or LP1 cells. However, expression of p53 was not affected by MIRA-1 treatment in these cells (Figure $3 \mathrm{~A}$ and $\mathrm{B}$ ) suggesting that activation of p53 may not require for MIRA-1-induced apoptosis of MM cells. To further confirm an involvement of p53 in MIRA1-induced apoptosis of MM cells, we next suppressed p53 expression by siRNA. In p53-silenced MM cells (Figure 3C), MIRA-1 was still able to induce apoptosis as judged by increase of Annexin V-positive cells (Figure 3D). These results suggest that 
A

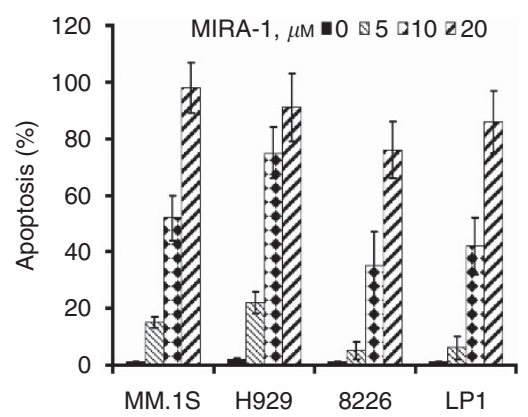

B

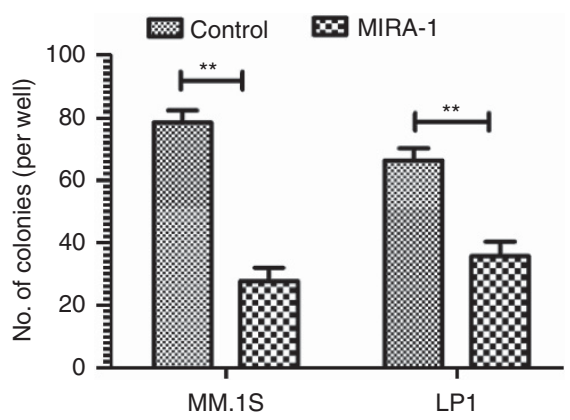

C

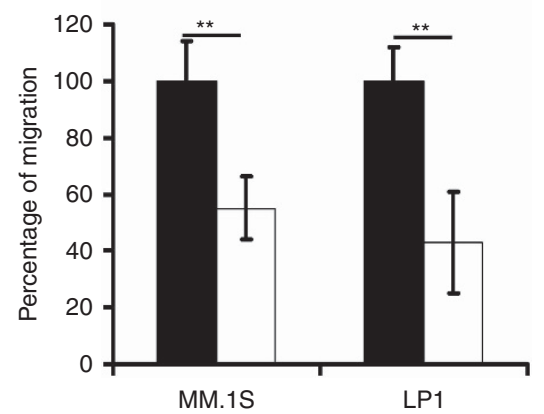

Figure 2. MIRA-1 induced apoptosis of MM cells irrespective of p53 status. (A) MM.1S, H929, 8266, and LP1 cells were treated with MIRA-1 $\left(0-20 \mu \mathrm{moll}^{-1}\right)$ or DMSO for $48 \mathrm{~h}$. Apoptosis was measured by Annexin $\mathrm{V}$ and PI binding assay by flow cytometry. Apoptotic cells were quantified after normalising with DMSO treated cells. (B) MIRA-1 inhibits colony formation in MM cells. MM.1S or LP1 cells $\left(5 \times 10^{4}\right.$ cells ml $\left.{ }^{-1}\right)$ treated with MIRA-1 or DMSO were used in forming cell layer in methyl cellulose-based medium. Colonies were counter 14 days later. (C) MIRA-1 inhibits the migration of MM cells. MM.1S or LP1 cells treated with DMSO or MIRA-1 were analysed for their migration ability. The migration assay was performed using a 24-well transwell plate and the migration cells were quantified as described in Materials and Methods. Data are expressed as mean \pm S.D. of triplicate cultures. ${ }^{\star} P<0.05,{ }^{\star *} P<0.01$.

p53 may not have a direct role in MIRA-1-induced apoptosis of MM cells.

To understand the molecular mechanisms underlying the observed MIRA-1-induced inhibition of cell growth, the expression of several intracellular regulators of the apoptosis was assessed by western blot analysis. MIRA-1 exposure resulted in an increase of expression for proapoptotic $\mathrm{BH} 3$ only proteins, Puma and Bax and a decrease of anti-apoptotic proteins, Mcl-1 and c-Myc (Figure 3A and B). In addition, MM.1S or LP1 cells treated with MIRA-1 exhibited time-dependent activation of PERK, calnexin, protein disulfide isomerase (PDI) (an oxidoreductase), and BIP (GRP78, a molecular chaperone) which represents stress-associated gene expression alterations. Examination of activation state of PERK and eIF2- $\alpha$ showed a sustained increase in the activating phosphorylation of both kinases that appeared as early as $4 \mathrm{~h}$ and persisted for 8-12 h (Figure 4A). Furthermore, splicing of XBP1 was observed by western blots (Figure 4A) and PCR analysis which was validated with a well-characterised endoplasmic reticulum (ER) stress-inducing agent, thapsigargin (Figure 4B). Our results suggest that MIRA-1-induced apoptosis in MM cells is associated with ER stress response signalling. On the basis of our observation, we depicted a diagram for the mechanism of action of MIRA-1 in MM cells (Figure 4C).

MIRA-1 enhances cytotoxicity of conventional and novel therapies. As novel anti-cancer agents are generally applied in combination with existing therapeutics, we examined whether MIRA-1 could potentiate the action of drugs currently used to treat MM. To this aim, MM cell lines or a primary MM sample were treated with combinations of MIRA-1 with either conventional drugs (dexamethasone or doxorubicin) or with novel antimyeloma agents (velcade). The cytotoxicity of the cells was analysed by MTT assays. As shown in Figure 5, simultaneous treatment of $8226 \mathrm{MM}$ cell line or a patient sample with MIRA-1 and dexamethasone or doxorubicin resulted in a significant decrease in cell survival when compared with the single agents $(P<0.05)$. When combined with low concentrations of these drugs, synergistic effects were observed $(\mathrm{CI}<1.0)$ (Figure $5 \mathrm{~A}$ and $\mathrm{B})$. We next assessed the ability of MIRA-1 to synergise with velcade. In both MM.1S and U266 cells, a significant decrease in the viability was observed in combination treatment compared with single treatment after $48 \mathrm{~h}$ of exposure of low doses of these drugs. The synergy was observed for these combinations with CI of lower than 1.0 (Figure 5C). These data demonstrated that combined treatment with equipotent doses of
MIRA-1 and dexamethasone, doxorubicin, or velcade resulted in synergistic effects in MM cells.

MIRA-1 effectively reduces tumour burden in a MM xenograft model. Next, we sought to examine whether in vitro MIRA-1induced MM cytotoxicity can be translated into effective in vivo anti-MM activity in SCID mice xenografted 8226 cells. Treatment with MIRA-1 significantly retarded tumour growth, with maximum tumour growth inhibition noted at day 15 (Figure 6A) $(P<0.05)$. MIRA-1 treatment was also associated with improved survival, evidenced by first death at day 20 in control versus day 45 in treated group (Figure $6 \mathrm{~B})(P=0.007)$. There were no obvious toxic effects of the treatments as evaluated by mouse body weight data (Figure 6C).

Finally, to test if enhanced myeloma cell apoptotic response is observed in vivo, we examined the efficacy of combination of MIRA-1 and dexamethasone in similar mouse xenograft model as described above. For combination studies, lower doses of MIRA-1 $\left(5 \mathrm{mg} \mathrm{kg}^{-1}\right)$ and dexamethasone $\left(1 \mathrm{mg} \mathrm{kg}^{-1}\right)$ were used. These lower concentrations of the drugs were chosen to reduce any potential for overlapping toxicities and side effects of these agents. Combination treatment with MIRA-1 and dexamethasone proved very efficacious as shown by significant inhibition of tumour growth compared with low dose of MIRA-1 or dexamethasone alone or vehicle (on day $15,{ }^{\star} P<0.05$ ) (Figure 6D). Importantly, as shown in Figure 6E, combination of MIRA-1 and dexamethasone resulted in a significant prolonged survival when compared with the vehicle group $(P=0.003)$, the dexamethasone-alone-treated group $(P=0.045)$ and MIRA-1-alone-treated group $(P=0.005)$. In addition, treatment with MIRA- 1 and/or dexamethasone did not affect body weight (Figure 6F) indicating the doses used for the treatment were tolerable to the mice. These data clearly shows that the combination of MIRA-1 and dexamethasone results in improvements of tumour burden and increased overall survival of the mice.

\section{DISCUSSION}

Small molecules for use in cancer treatment have been in the forefront of developmental therapeutics in recent years. Most clinically evaluated small molecules targeting p53 exert their antitumour activities in tumour cells harbouring either wild-type or mutant p53 (Bykov et al, 2002; Issaeva et al, 2004; Bykov et al, 2005; 
A

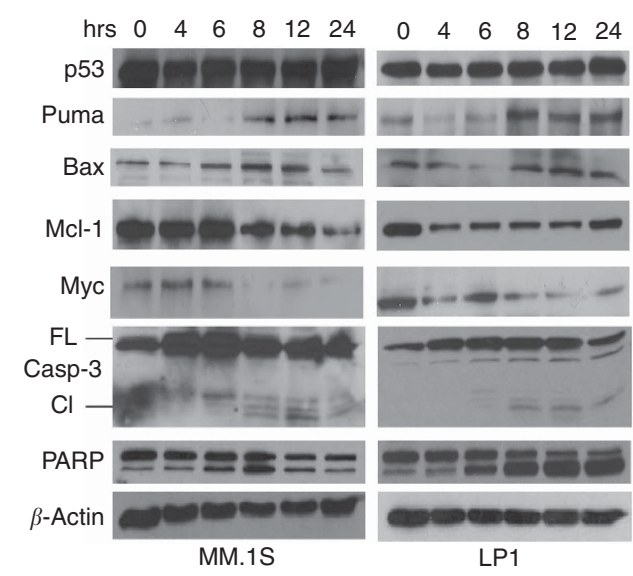

B

MIRA-1, $\mu$ mol I $^{-1} 0 \quad 10 \quad 20$

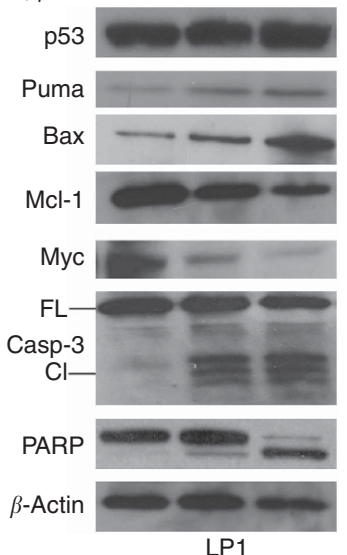

C

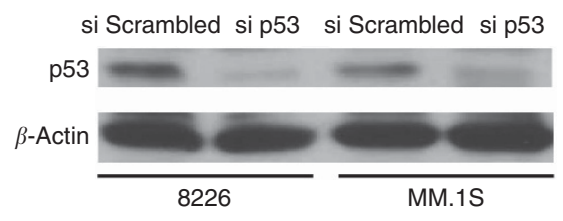

D $\quad$ si Scrambled

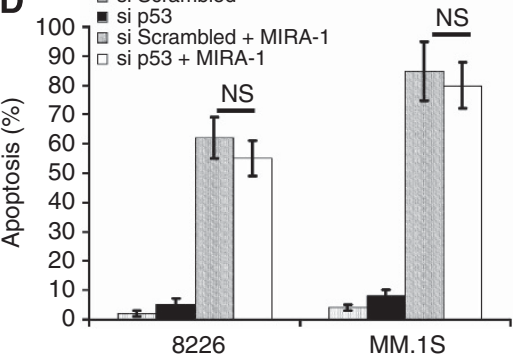

Figure 3. MIRA-1 induced time and dose-dependent apoptosis in MM cells. MIRA-1 treatment results in upregulation of Puma and Bax and downregulation of Mcl-1 and c-Myc in a time- and dose-dependent manner with associated activation of caspase-3 and PARP. (A) Total levels of the indicated proteins were evaluated by western blot analysis in the indicated cell lines after treatment with $10 \mu \mathrm{molI}{ }^{-1}(\mathrm{MM} .1 \mathrm{~S})$ or $20 \mu \mathrm{mol} \mathrm{I}^{-1}$ (LP1) cells for up to $24 \mathrm{~h}$. (B) LP1 cells were treated with indicated concentrations of MIRA-1 for $8 \mathrm{~h}$. Total cell lysates were prepared and analysed by western blot for the expression of the indicated protein. (C and D) p53 siRNA-transfected MM.1S and 8226 cells were further treated with 10 or $20 \mu \mathrm{moll}^{-1}$ MIRA-1, respectively, for $48 \mathrm{~h}$ and analysed for apoptosis induction by FCM; NS, not significant.

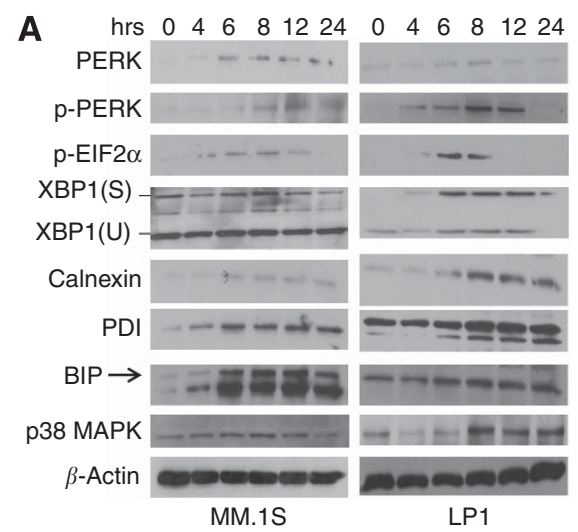

B

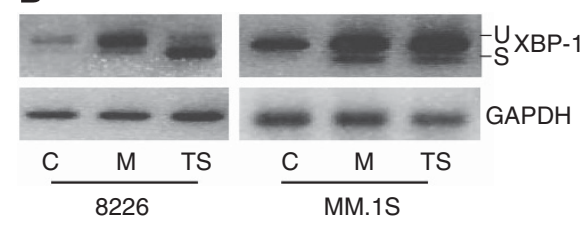

\section{C}
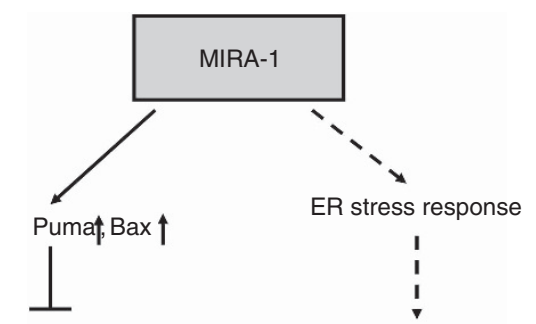

c-Myc, Mcl-1 $\downarrow$ :- - - - PERK/e-IF2 $\alpha \uparrow$

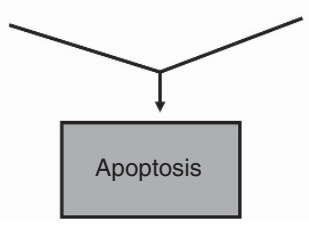

Figure 4. MIRA-1 induced an ER stress response in MM cells. (A) MM.1S and LP1 cells were treated with MIRA-1 for $8 \mathrm{~h}$, total proteins were harvested and analysed by Western blot for the expression of the indicated proteins. (B) XBP1 mRNA splicing by MIRA-1. RT-PCR analysis with XBP1 or GAPDH-specific primers of total RNA extracted from MM.1S or 8226 cells that were incubated with 10 or $20 \mu$ mol I-1 MIRA-1, respectively, for $8 \mathrm{~h}$ as indicated. XBP1U = unspliced XBP1; XBP1S = spliced XBP1. Thapsigargin (TS) $\left(100 \mathrm{nmol} \mathrm{I}^{-1}\right)$ was used as a positive control ER stress-inducing agent. (C) Proposed model for the p53-independent apoptotic function of MIRA-1 in MM cells. MIRA-1 may directly interact with mitochondria function inhibiting Bcl2 family members Mcl-1 and/or Myc through upregulation of Puma and Bax. Alternatively, inhibition of Mcl-1 by MIRA-1 may be indirectly mediated by activation of ER stress signalling. 

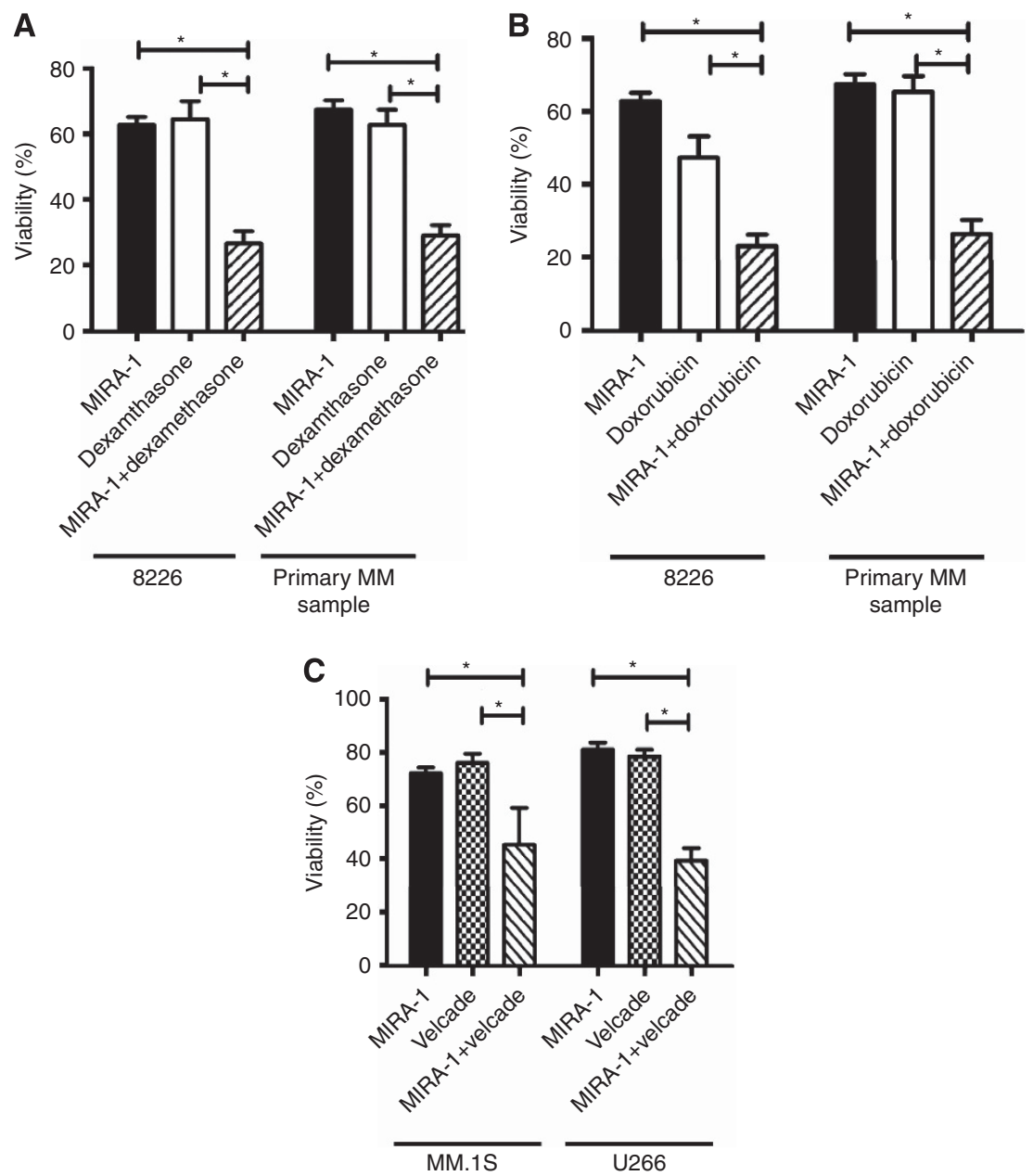

Figure 5. MIRA-1 in combination with current anti-myeloma agents displayed synergistic cytotoxic response in MM cells. Cells obtained from either cell lines or patient samples were treated with MIRA-1 $\left(10 \mu \mathrm{molI} \mathrm{I}^{-1}\right)$ in combination with $(\mathbf{A})$ dexamethasone $\left(1.0 \mu \mathrm{mol} \mathrm{I}^{-1}\right)$ or (B) doxorubicin $\left(1.0 \mu \mathrm{moll} \mathrm{I}^{-1}\right)$. (C) MM.1S or U266 cells were treated with MIRA-1 $\left(5 \mu \mathrm{moll}^{-1}\right)$ and velcade $\left(2.5 \mathrm{nmoll} \mathrm{I}^{-1}\right) .48 \mathrm{~h}$ after treatment, cells were assessed for survival by MTT viability assay $\left(^{*}<0.05\right)$. Data are expressed as mean \pm s.d. of triplicate cultures of at least two independent experiments.

Wang and El-Deiry 2008; Saha et al, 2010a, b, c, d). However, recent reports indicate that some of these small molecules, initially specified for working on wild-type or mutant p53, can also kill cancer cells independent of p53 status or even in the absence of p53 (Cory et al, 2006; Supiot et al, 2008; Zhao et al, 2010; Bao et al, 2011; Wang et al, 2011; Burmakin et al, 2013; Saha et al, 2013a, b)

MIRA-1 was initially identified as a mutant p53 reactivating compound capable of restoring wild-type conformation of mutant p53. We evaluated anti-tumour activities of MIRA-1 in MM cells with varying p53 status. Unlike the previous study, MIRA-1 exposure in MM cells resulted in significant cytotoxicity in both wild-type and dysfunctional p53. Previous studies described that MIRA-1 induced transcriptional transactivation of p53 through a sequence-specific binding. Our results confirm the previous observations that MIRA-1 restores wild-type conformation of mutant p53 (data not shown). However, in contrast to the previous report, our studies on wild-type, mutant or silenced p53 MM cells showed that p53 status was not a determinant of the effect of MIRA-1 on apoptosis in MM cells.

These contradictory but interesting findings prompted us to investigate the potential mechanisms of MIRA-1-induced cytotoxicity in MM cells. In this study, we demonstrated that MIRA-1-induced apoptosis in MM cells was, at least in part, p53-independent as genetic knockdown of p53 did not have significant impact on apoptosis induction by MIRA-1. However, the role of p53 in MIRA-1-induced apoptosis of MM cells cannot be fully excluded. This is due to the fact that transcripts of p53 and its downstream targets Puma and Bax were induced by MIRA-1. This is in agreement with previous studies on osteosarcoma cells (Bykov et al, 2005). Interestingly, p53-independent upregulation of Puma and Bax has been shown previously in other cancer types (Jeffers et al, 2003; Gogada et al, 2011). Along with increased pro-apoptotic Puma and Bax, MIRA-1 decreased anti-apoptotic proteins Mcl-1 and c-Myc in MM cells. Similar to our studies, prior studies in MM have also linked the downregulation of Mcl-1 and Myc to apoptotic pathway (Gojo et al, 2002; Gauduchon et al, 2005; Gomez-Bougie et al, 2007). Importantly, both Mcl-1 and $\mathrm{c}-\mathrm{Myc}$ are considered key regulators of cell growth and protein synthesis. Moreover, overexpression of Mcl-1 and/or c-Myc has been proposed to be associated with progression of MM (Zhang et al, 2002; Holien et al, 2012). Although, several mechanisms have been proposed to p53-dependent apoptosis, very little is known about the p53-independent pathways. Therefore, the study of alternative pathways activated by MIRA-1 may be relevant to rescue an apoptotic response in cells lacking p53 function.

In this study, using qPCR array we identified a set of key genes in p38MAPK signalling pathway that are differentially expressed in two types of MM cell lines treated with MIRA-1 (data not shown). 
A

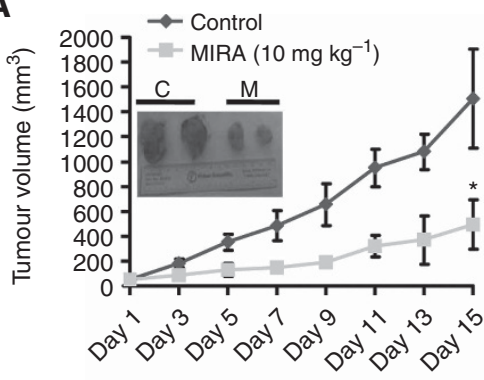

Day after injection

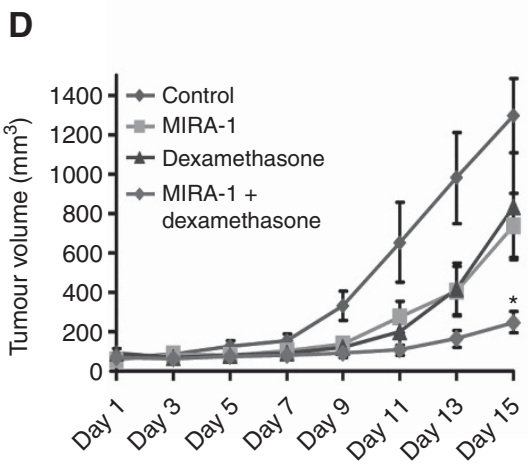

Day after injection
B

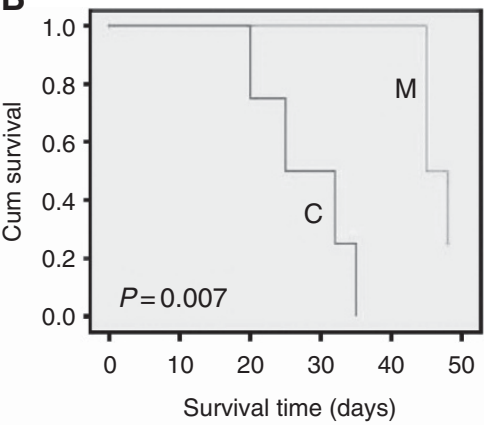

E

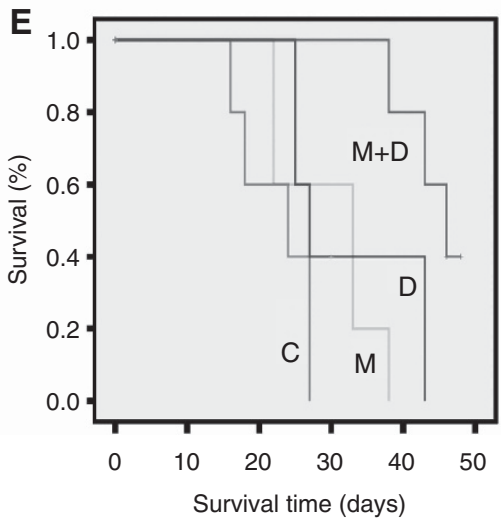

C

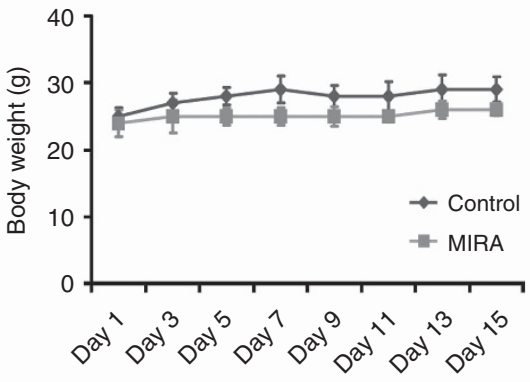

$\mathbf{F}$

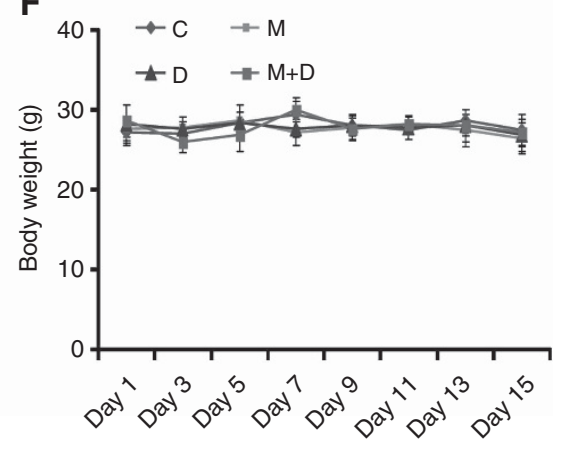

Day after injection

Figure 6. MIRA-1 induced anti-myeloma activity in vivo. SCID mice ( $n=5$ per group) were inoculated s.c. with $3 \times 10^{7} 8226$ cells in RPMI medium along with matrigel matrix. Tumour-bearing mice were randomly assigned into two cohorts receiving daily i.p. injection of MIRA-1 $\left(10 \mathrm{mg} \mathrm{kg}^{-1}\right.$ ) or with vehicle (PBS) alone for 15 days. (A) MIRA-1 treatment resulted in tumour growth inhibition ( $\left.{ }^{\star} P<0.05\right)$. (B) Prolonged survival was observed in the treatment group of mice $(P=0.007)$. (C) No significant loss of body weight was observed. (D-F) MIRA-1 enhanced anti-tumour activity of dexamethasone in vivo. SCID mice were inoculated s.c. with $3 \times 10^{7} 8226$ cells in $100 \mu \mathrm{l}$ RPMI medium together with matrigel matrix. Twenty tumour-bearing mice were randomly assigned to four groups and treated for 13 days with control (PBS), MIRA-1 (5 mg kg ${ }^{-1}$ ), dexamethasone $\left(1 \mathrm{mg} \mathrm{kg}^{-1}\right)$, or the combination once daily. At day 15 , the treatment was discontinued and mice were monitored for tumour dynamics and body weight. (D) MIRA-1 and dexamethasone combination therapy triggered more potent inhibition of tumour growth in mice treated with MIRA-1 or dexamethasone alone or control ( $\left.{ }^{*}<<0.05\right)$. (E) Combination therapy markedly prolonged survival compared with mice treated with control $(P=0.003)$, MIRA-1 $(P=0.005)$ or dexamethasone $(P<0.045)$ alone. $(F)$ Mice body weight was used to assess toxicity of the treatment. $C=$ control; $M=$ MIRA-1; $D=$ dexamethasone; $M+D=$ MIRA-1 + dexamethasone.

Many of these genes have been involved in apoptosis/cell proliferation, DNA binding, signal transduction, and transcription regulation indicating that MIRA-1 activates multiple signalling pathways. For selected genes, we have further confirmed the observed changes in GPCR array at protein levels. Both gene expression and protein data showed the activation of p38 MAPK signalling which is a common consequence of ER stress response. Moreover, MIRA-1 induced activation of at least one of the ER stress sensors PERK which leads to its phosphorylation as well as phosphorylation of eIF2- $\alpha$ and activation of a pool of molecular chaperone proteins including calnexin, PDI, and Bip. These results together with XBP1 splicing indicate that MIRA-1 treatment is associated with induction of ER stress signalling. Association of ER stress response in p53-independent apoptosis of cancer cells have previously been reported (Gomez-Lazaro et al, 2008; Jiang et al, 2008). Notably, ER stress signalling has been shown to be linked with the activation of Puma/Bax/Noxa or repression of Mcl-1 signalling axis (Li et al, 2006; Chauhan et al, 2011). Our data correlates with the activation of Puma/Bax, Mcl-1/Myc, and ER stress signalling pathways and suggest a potential cross-talk between these pathways during MIRA-1-induced apoptosis of MM cells (Figure 4C). Although the mechanism of MIRA-1induced apoptosis in not full defined, these effects are likely dependent on the ER stress response. Further studies will be required to understand how MIRA-1 modulates intracellular signalling molecules leading to apoptosis and the role of the specific signalling molecules associated with MIRA-1-induced apoptosis.

\section{CONCLUSIONS}

MIRA-1 treatment resulted in a potent anti-myeloma activity, both in vitro and in vivo, and leads to induction of multiple interrelated pathways implicated in apoptosis of MM cells. MIRA-1 induces p53-independent apoptosis in MM cells and the apoptotic response is closely related to the changes in the balance between proapoptotic and antiapoptotic Bcl2 family proteins favouring induction of apoptosis. Added potential includes its synergy with current myeloma therapeutics. Our results suggest that the use of MIRA-1 either alone or in combination with other therapeutic agents is worth considering for further clinical evaluation in MM.

\section{ACKNOWLEDGEMENTS}

This study was supported by grants in part from Leukemia \& Lymphoma Society, Canada, Cancer Research Society, International Collaboration Fund from Chinese Ministry of Science and Technology (No. 2011DFA32820), and Gan-Po 555 project, Jiangxi, China awarded to H Chang. 


\section{CONFLICT OF INTEREST}

The authors declare no conflict of interest.

REFERENCES

Anderson KC (2011) Oncogenomics to target myeloma in the bone marrow microenvironment. Clin Cancer Res 17: 1225-1233.

Bao W, Chen M, Zhao X, Kumar R, Spinnler C, Thullberg M, Issaeva N, Selivanova G, Strömblad S (2011) PRIMA-1Met/APR-246 induces wild-type p53-dependent suppression of malignant melanoma tumor growth in 3D culture and in vivo. Cell Cycle 10: 301-307.

Burmakin M, Shi Y, Hedström E, Kogner P, Selivanova G (2013) Dual targeting of wild-Type and mutant p53 by small molecule RITA results in the inhibition of N-Myc and key survival oncogenes and kills neuroblastoma cells in vivo and in vitro. Clin Cancer Res 19: 5092-5103.

Bykov VJ, Issaeva N, Shilov A, Hultcrantz M, Pugacheva E, Chumakov P, Bergman J, Wiman KG, Selivanova G (2002) Restoration of the tumor suppressor function to mutant p53 by a low-molecular-weight compound. Nat Med 8: 282-288.

Bykov VJ, Issaeva N, Zache N, Shilov A, Hultcrantz M, Bergman J, Selivanova G, Wiman KG (2005) Reactivation of mutant p53 and induction of apoptosis in human tumor cells by maleimide analogs. J Biol Chem 280: 30384-30391.

Chang H, Qi C, Yi Q, Reece D, Stewart AK (2005) p53 gene deletion detected by fluorescence in situ hybridization is an adverse prognostic factor for patients with multiple myeloma following autologous stem cell transplantation. Blood 105: 358-360.

Chauhan D, Tian Z, Zhou B, Kuhn D, Orlowski R, Raje N, Richardson P, Anderson KC (2011) In vitro and in vivo selective antitumor activity of a novel orally bioavailable proteasome inhibitor MLN9708 against multiple myeloma cells. Clin Cancer Res 17: 5311-5321.

Cory AH, Chen J, Cory JG (2006) Effects of PRIMA-1 on wild-type L1210 cells expressing mutant p53 and drug-resistant L1210 cells lacking expression of p53: necrosis vs. apoptosis. Anticancer Res. 2006 26: 1289-1295.

Dimopoulos MA, San-Miguel JF, Anderson KC (2011) Emerging therapies for the treatment of relapsed or refractory multiple myeloma. Eur J Haematol 86: $1-15$.

Gogada R, Prabhu V, Amadori M, Scott R, Hashmi S, Chandra D (2011) Resveratrol induces p53-independent, X-linked inhibitor of apoptosis protein (XIAP)-mediated Bax protein oligomerization on mitochondria to initiate cytochrome $\mathrm{c}$ release and caspase activation. J Biol Chem 286: 28749-28760.

Gojo I, Zhan B, Fenton RG (2002) The cyclin-dependent kinase inhibitor flavopiridol induces apoptosis in multiple myeloma cells through transcriptional repression and down-regulation of Mcl-1. Clin Cancer Res 8: 3527-3538.

Gomez-Bougie P, Wuillème-Toumi S, Ménoret E, Trichet V, Robillard N, Philippe M, Bataille R, Amiot M (2007) Noxa up-regulation and Mcl-1 cleavage are associated to apoptosis induction by bortezomib in multiple myeloma. Cancer Res 67: 5418-5424.

Gomez-Lazaro M, Galindo MF, Concannon CG, Segura MF, Fernandez-Gomez FJ, Llecha N, Comella JX, Prehn JH, Jordan J (2008) 6-Hydroxydopamine activates the mitochondrial apoptosis pathway through p38 MAPK-mediated, p53-independent activation of Bax and PUMA. J Neurochem 104: 1599-1612.

Gauduchon J, Gouilleux F, Maillard S, Marsaud V, Renoir JM, Sola B (2005) 4-Hydroxytamoxifen inhibits proliferation of multiple myeloma cells in vitro through down-regulation of c-Myc, up-regulation of p27Kip1, and modulation of Bcl-2 family members. Clin Cancer Res 11: 2345-2354.

Holien T, Våtsveen TK, Hella H, Waage A, Sundan A (2012) Addiction to c-MYC in multiple myeloma. Blood 120: 2450-2453.

Issaeva N, Bozko P, Enge M, Protopopova M, Verhoef LG, Masucci M, Pramanik A, Selivanova G (2004) Small molecule RITA binds to p53, blocks p53-HDM-2 interaction and activates p53 function in tumours. Nat Med 12: 1321-1328.

Jeffers JR, Parganas E, Lee Y, Yang C, Wang J, Brennan J, MacLean KH, Han J, Chittenden T, Ihle JN (2003) Puma is an essential mediator of p53-dependent and -independent apoptotic pathways. Cancer Cell 4: 321-328.

Jiang CC, Lucas K, Avery-Kiejda KA, Wade M, deBock CE, Thorne RF, Allen J, Hersey P, Zhang XD (2008) Up-regulation of Mcl-1 is critical for survival of human melanoma cells upon endoplasmic reticulum stress. Cancer Res 68: 6708-6717.

Li J, Lee B, Lee AS (2006) Endoplasmic reticulum stress-induced apoptosis: multiple pathways and activation of p53-up-regulated modulator of apoptosis (PUMA) and NOXA by p53. J Biol Chem 281: 7260-7270.

Mitsiades CS, Hideshima T, Chauhan D, McMillin DW, Klippel S, Laubach JP, Munshi NC, Anderson KC, Richardson PG (2009) Emerging treatments for multiple myeloma: beyond immunomodulatory drugs and bortezomib. Semin Hematol 46: 166-175.

Mazars GR, Portier M, Zhang XG, Jourdan M, Bataille R, Theillet C, Klein B (1992) Mutations of the p53 gene in human myeloma cell lines. Oncogene 7: $1015-1018$.

Saha MN, Micallef J, Qiu L, Chang H (2010a) Pharmacological activation of the p53 pathway in haematological malignancies. J Clin Pathol 63: 204-209.

Saha MN, Jiang H, Jayakar J, Reece D, Branch DR, Chang H (2010b) MDM2 antagonist nutlin plus proteasome inhibitor velcade combination displays a synergistic anti-myeloma activity. Cancer Biol Ther 9: 936-944.

Saha MN, Jiang H, Chang H (2010c) Molecular mechanisms of nutlin-induced apoptosis in multiple myeloma: evidence for p53-transcription-dependent and -independent pathways. Cancer Biol Ther 2010 10: 567-578.

Saha MN, Jiang H, Muaki A, Chang H (2010d) RITA inhibits multiple myeloma cell growth through induction of p53-mediated caspase-dependent apoptosis and synergistically enhances nutlin-induced cytotoxic responses. Mol Cancer Ther 9: 3041-3051.

Saha MN, Jiang H, Yang Y, Zhu X, Wang X, Schimmer AD, Qiu L, Chang H (2012) Targeting p53 via JNK Pathway: a novel role of RITA for apoptotic signaling in multiple myeloma. PLoS One 2012 7: e30215.

Saha MN, Qiu L, Chang H (2013a) Targeting p53 by small molecules in hematological malignancies. J Hematol Oncol 6: 23.

Saha MN, Jiang H, Yang Y, Reece D, Chang H (2013b) PRIMA-1Met/APR-246 displays high anti-tumor activity in multiple myeloma by induction of p73 and Noxa. Mol Cancer Ther 12: 2331-2341.

Supiot S, Zhao H, Wiman K, Hill RP, Bristow RG (2008) PRIMA-1(met) radiosensitizes prostate cancer cells independent of their MTp53-status. Radiother Oncol 86: 407-411.

Vassilev LT, Vu BT, Graves B, Carvajal D, Podlaski F, Filipovic Z, Kong N, Kammlott U, Lukacs C, Klein C et al. (2004) In vivo activation of the p53 pathway by small-molecule antagonists of MDM2. Science 303: 844-848.

Wang W, El-Deiry WS (2008) Restoration of p53 to limit tumor growth. Curr Opin Oncol 20: 90-96.

Wang J, Zheng T, Chen X, Song X, Meng X, Bhatta N, Pan S, Jiang H, Liu L (2011) MDM2 antagonist can inhibit tumor growth in hepatocellular carcinoma with different types of p53 in vitro. Gastroenterol Hepatol. 2011 26: 371-377.

Wiman KG (2006) Strategies for therapeutic targeting of the p53 pathway in cancer. Cell Death Differ 13: 921-926.

Zhang B, Gojo I, Fenton RG (2002) Myeloid cell factor-1 is a critical survival factor for multiple myeloma. Blood 99: 1885-1893.

Zhao CY, Grinkevich VV, Nikulenkov F, Bao W, Selivanova G (2010) Rescue of the apoptotic-inducing function of mutant p53 by small molecule RITA. Cell Cycle 9: 1847-1855.

This work is published under the standard license to publish agreement. After 12 months the work will become freely available and the license terms will switch to a Creative Commons AttributionNonCommercial-Share Alike 3.0 Unported License. 